Ann. Biol. anim. Bioch. Biophys., I976, 16 (5), 675-686.

\title{
ÉVOLUTION PÉRINATALE DES VOIES MÉTABOLIQUES GLYCOLYTIQUE ET OXYDATIVE DE DIVERS TYPES DE MUSCLES SQUELETTIQUES DU LAPIN ET DU POULET
}

\author{
F. BACOU et P. VIGNERON \\ Station de Physiologie animale, \\ Centre de Recherches de Montpellier, I. N. R. A., E. N. S. A., \\ Place Viala, \\ 34060 Montpellier Cedex
}

RÉSUMÉ

Au cours de ce travail, nous avons suivi chez le Lapin et le Poulet l'évolution périnatale des métabolismes glycolytique et oxydatif dans plusieurs muscles choisis en fonction de leurs caractéristiques chez l'animal adulte. Celui-ci possède une musculature complètement différenciée, où l'on distingue les trois types de fibres musculaires ( $\beta R, \alpha R$ et $\alpha W$ ) et des profils enzymatiques caractéristiques d'un muscle donné. Au cours de la différenciation musculaire, que ce soit chez le Lapin ou le Poulet, le métabolisme des muscles "blancs ", oxydatif à la naissance, évolue vers un métabolisme nettement glycolytique à l'âge adulte ; les muscles de types " rouges ", par contre, ne semblent pas présenter de telles transformations, leur métabolisme étant toujours à prépondérance oxydative.

L'évolution d'enzymes caractérisant ces deux métabolismes est plus ou moins complexe selon l'espèce et le type de muscle considérés : l'activité aldolasique du muscle " blanc " cruît progressivement chez le Lapin, brutalement entre $\mathbf{5}$ et ro jours chez le Poulet, tandis qu'elle demeure faible et stationnaire dans les muscles " rouges ". L'activité des enzymes oxydatives passe par un maximum au cours des 30 premiers jours postnatals, le niveau de l'adulte étant inférieur à celui mesuré à la naissance quels que soient l'espèce animale ou les types de muscles étudiés.

\section{INTRODUCTION}

Les propriétés dynamiques (BULLER, ECcLES et al., I96o, I965) et les capacités métaboliques des muscles squelettiques des vertébrés adultes (PREWITT et SALAFSKy, I967; I)Ubowiтz, I967 ; RoManul et al., I 967, I97I) résultent de leurs carac- 
téristiques structurales, c'est-à-dire de leurs richesses relatives en l'un ou l'autre des divers types de fibres musculaires. Celles-ci peuvent être rangées en trois catégories principales (ASHMORE et al., I972) : fibres à contraction rapide et métabolisme glycolytique $(\alpha W)$, fibres à contraction rapide et métabolisme oxydatif $(\alpha R)$, fibres à contraction lente et métabolisme oxydatif $(\beta R)$. Le nombre et le diamètre des fibres, leurs propriétés métaboliques et enzymatiques conditionnent la composition biochimique du tissu musculaire et influent notamment sur les caractéristiques technologiques et organoleptiques des viandes.

A la suite des travaux de Close (I964) et de Cosmos (1966, I970), les fibres musculaires apparaissent essentiellement de type oxydatif à la naissance, la différenciation métabolique s'effectuant principalement au cours du développement. Au cours de ce travail, nous avons suivi l'évolution périnatale du métabolisme glycolytique et oxydatif dans plusieurs muscles choisis en fonction de leurs caractéristiques chez l'adulte. Deux espèces animales ont été retenues, très différentes quant à l'état de maturité physiologique des jeunes à la naissance : le Lapin et le Poulet, ce dernier permettant des investigations plus appronfondies au cours de la période embryonnaire.

\section{MATÉRIEL ET MÉTHODES}

Les animaux issus des élevages du laboratoire (lapins Néo-Zélandais, poulets Nere-Hampshive $\mathrm{C}_{2}$ ) ont été étudiés au cours de la croissance aux âges précisés sur les figures 4 et 5 . Deux sortes d'études, histochimique et enzymatique, ont été effectuées sur différents tissus musculaires, prélevés dès le sacrifice des animaux et analysés immédiatement.

\section{A. - Étude histologique}

Elle a été réalisée sur des muscles d'animaux adultes. La révélation de l'activité de la succinate déhydrogénase (NACHLAS, 1955) et de l'adénosine triphosphatase myofibrillaire après préincubation alcaline (PADYkUla et Herman, 1955) ou acide (BROOKE et KaISER, I969) a permis de caractériser et de choisir les muscles suivants pour l'étude enzymatique : lytique).

- Chez le Lapin, le soleus (muscle lent, oxydatif) et le psoas major (muscle rapide, glyco-

- Chez le Poulet, les muscles ont été groupés selon leur type de métabolisme doninant : pestoralis major, supracoracoideus, latissimus dorsi posterior (glycolytiques), latissimus dorsi anterior, adductor magnus, adductor brevis, flexor digitus perforatus digiti (oxydatifs). L'évolution des activités enzymatiques a été suivie chez le Poulet sur deux groupes de ces muscles : pectoralis major, supracoracoideus d'une part, et adductor magnus, adductor brevis d'autre part.

\section{B. - Étude enzymatique}

Les caractéristiques principales de la différenciation métabolique peuvent être déterminées par l'étude des schémas d'activité enzymatique (Petre, 197I). Pour caractériser le métabolisme glycolytique, nous avons adopté la fructose diphosphate aldolase (E.C. : 4-I-2-I 3), la voie oxydative étant représentée par la NADP isocitrate déhydrogénase (E.C. : I-I-I-42; ICDH) et la succinate déhydrogénase (E.C. : r-3-99-I ; SDH) chez le Lapin.

Ces enzymes ont été extraites selon la technique de Dawson et Romanul (I964) dans une solution de sucrose $0,25 \mathrm{M}$; les dosages ont été effectués en cinétique continue dans les cuvettes thermostatées $\left(37^{\circ} \mathrm{C}\right)$ d'un spectrophotomètre enregistreur (Gilford 240 ), en suivant à $34^{\circ} \mathrm{nm}$ les vitesses d'oxydation du NADH (aldolase) et de réduction du NADP (ICDH). Le dosage de l'activité SDH a été réalisé d'après la méthode de Slater et BonNer (I952) modifiée par AdLARD et DobBing (I97I). 


\section{RÉSULTATS}

L'état de différenciation métabolique du tissu musculaire dépend de l'âge de 1'animal étudié d'une part, et du type de muscle considéré d'autre part. L'animal adulte (défini ici comme ayant atteint le plateau de croissance) possède une musculature complètement différenciée, où l'on distingue les trois types de fibres musculaires (planche A) et des profils enzymatiques caractéristiques d'un muscle donné.

Chez le Lapin âgé de 6 mois (adulte), le soleus et le psoas major présentent une organisation métabolique différente (fig. I) : à une importante activité aldolasique dans le psoas se superpose une faible activité $\mathrm{SDH}$, le schéma de ces métabolismes étant inverse pour le soleus.

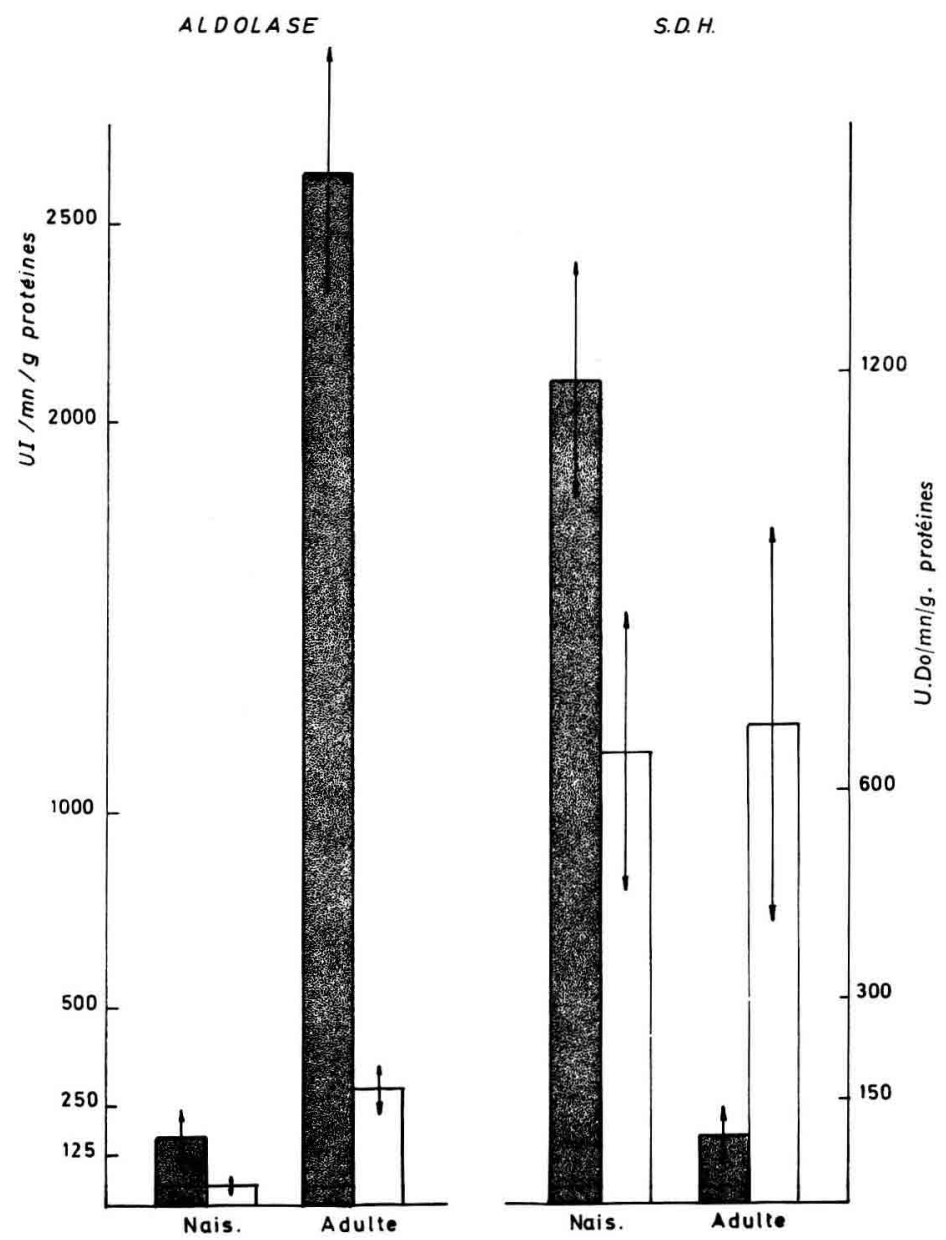

Fig. I. - Comparaison de l'activitê des deux enzymes étudiées dans les muscles psoas major et soleus chez le Lapin

Gris : Psoas

Blanc : Soleus 
Les sept muscles étudiés chez le Poulet de 20 semaines montrent des caractéristiques enzymatiques différentes, pouvant servir de base à l'établissement d'une classification selon la prépondérance du métabolisme glycolytique ou oxydatif (fig. 2 ). Les muscles pectoralis major, supracoracoideus et latissimus dorsi posterior ont une activité aldolasique importante et une activité ICDH faible : ce sont des muscles à métabolisme essentiellement anaérobie, composés d'une majorité de fibres $\alpha \mathrm{W}$, à contraction rapide; à l'opposé, les deux adductors, le latissimus dorsi anterior et le flexor digitus perforatus digiti ont un métabolisme à prépondérance aérobie : faible activité aldolasique, activité $I C D H$ nettement plus importante que dans les muscles précédents.
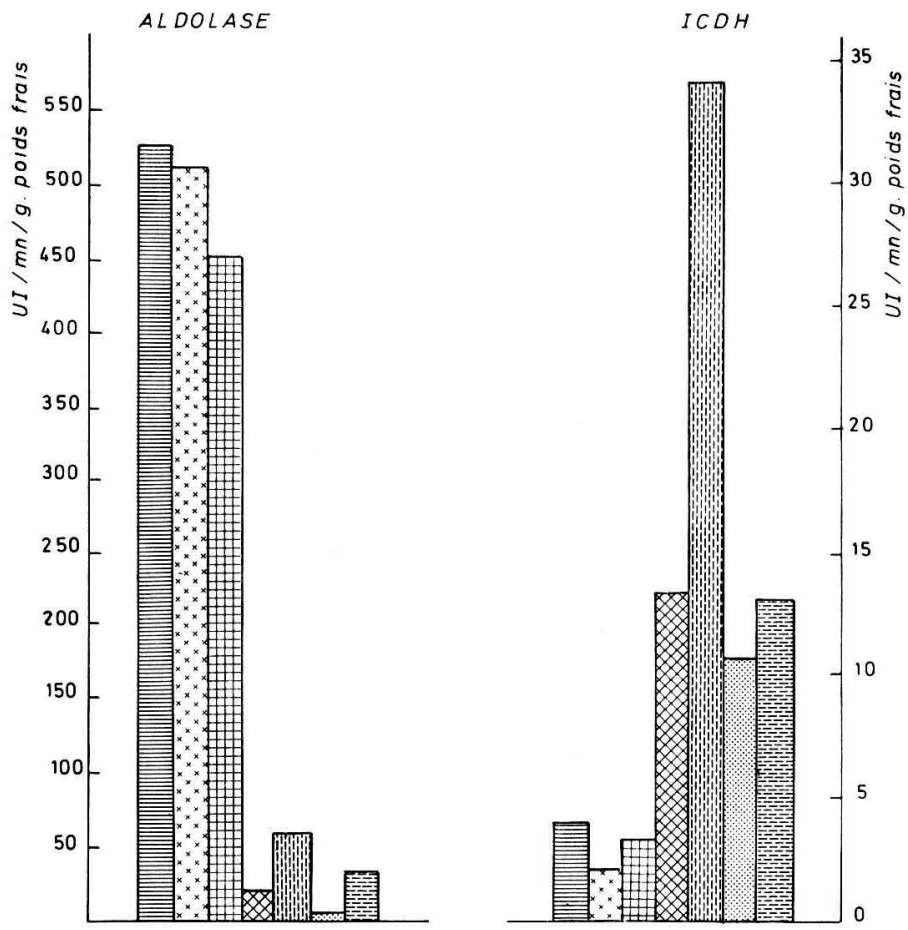

F1G. 2. - Activités enzymatiques chez plusieus muscles du paulet adulte

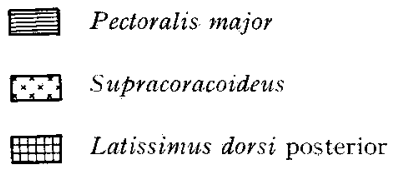

\author{
Latissimus dorsi anterior

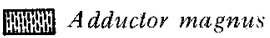 \\ Adductor brevis \\ Flexor digitus perforatus digiti
}

Pour atteindre cet état très différencié, les divers tissus musculaires subissent de multiples transformations qui se manifestent notamment par des variations d'activités enzymatiques décelables à la naissance et au cours de la période périnatale. 
A la naissance, chez le Lapin, les métabolismes des deux muscles considérés sont très différents de l'adulte : le psoas possède alors toutes les caractéristiques d'un muscle oxydatif avec une faible activité aldolasique et une activité SDH très importante, le schéma étant inverse chez l'adulte (fig. I) ; le soleus, par contre, ne présente pas les mêmes changements d'activité, l'activité aldolasique, bien qu'augmentant se situe à un niveau très bas par rapport au psoas, alors que l'activité SDH présente un niveau identique à la naissance et à six mois.

Chez le Poulet (fig. 3), le groupe des pectoralis présente à la naissance, comme nous l'avons observé pour le psoas du lapin, des caractéristiques de muscle adulte de type oxydatif. L'activité aldolasique des adductors évolue relativement peu par rapport à celle des pectoralis, alors que l'activité oxydative de 1'ICDH marque une chute importante. L'examen du métabolisme chez l'embryon de Io jours montre
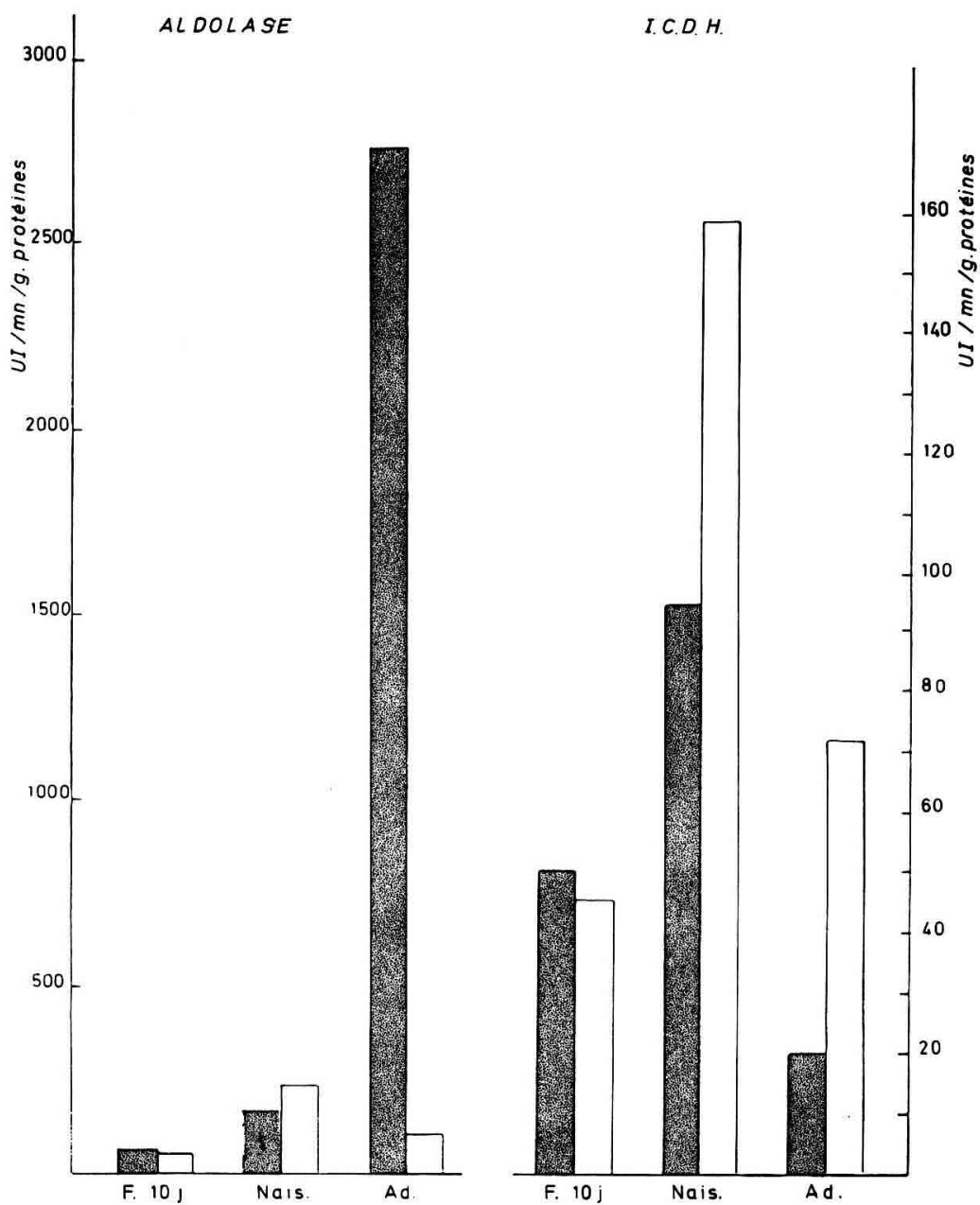

FIG. 3. - Aclivités enzymatiques des groupes Pectoralis et Adductors du poulet à différents âges

Gris : Pectoralis

Blanc : Adductors 
que les deux groupes de muscles ont des activités enzymatiques semblables. Il est intéressant de remarquer que l'activité oxydative ICDH est plus importante dans les pectoralis à cet âge embryonnaire que chez l'adulte.

Ainsi donc, que ce soit chez le Lapin ou le Poulet, les muscles de type "blanc" évoluent depuis la naissance jusqu'à l'âge adulte d'un métabolisme oxydatif vers un métabolisme nettement glycolytique ; les muscles de type " rouge ", par contre, ne semblent pas présenter de telles transformations, leur métabolisme étant toujours à prépondérance oxydative.

Pour situer au cours de la vie animale les principales transformations métaboliques, nous avons suivi dans ces mêmes groupes de muscles, au cours de la croissance, l'évolution des activités aldolasiques et SDH chez le Lapin à partir de la naissance jusqu'à six mois d'une part, ainsi que les modifications des activités de l'aldolase et de l'ICDH chez le Poulet depuis l'embryon de Io jours jusqu'à l'animal adulte (20 semaines) d'autre part.
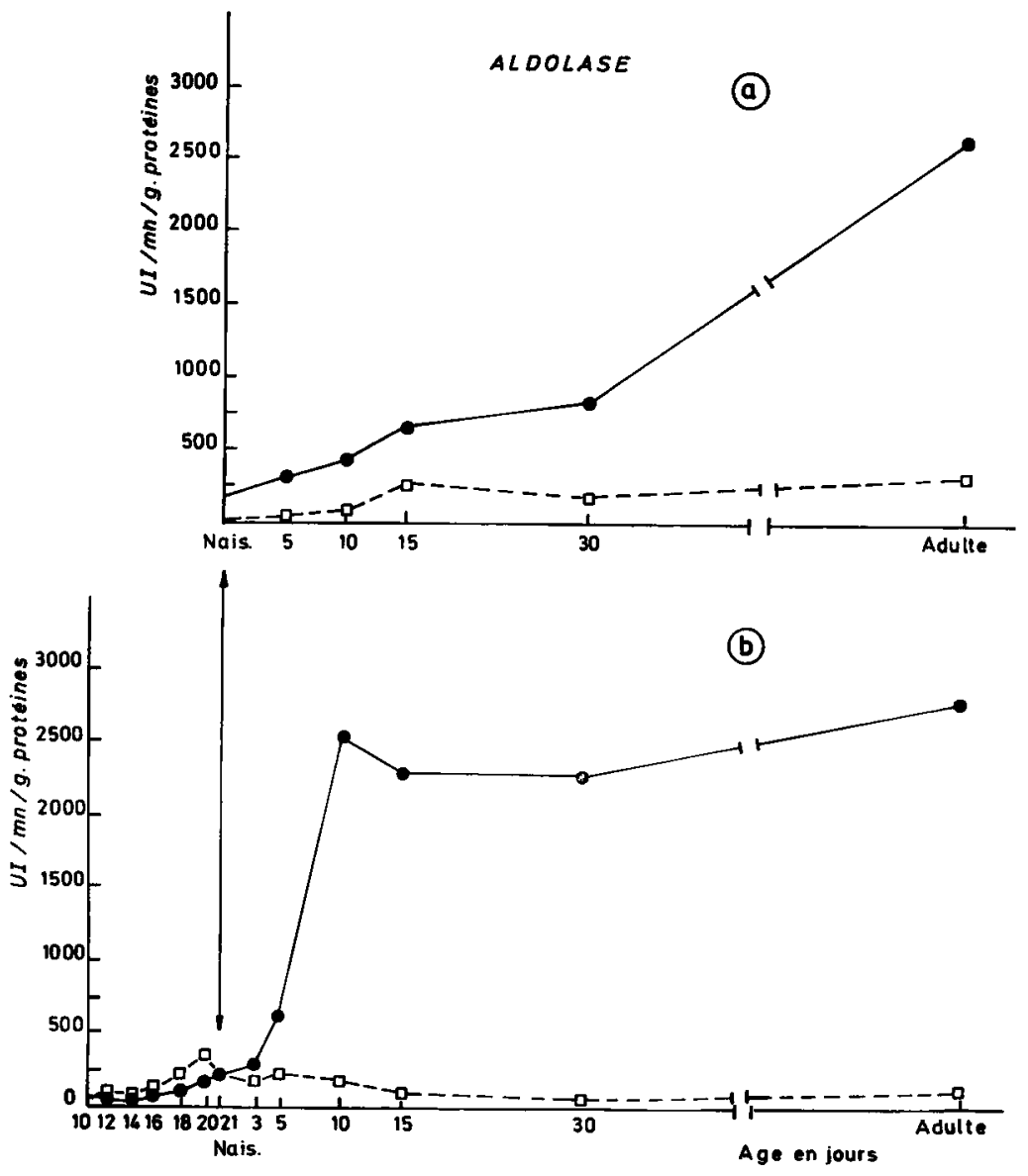

FIG. 4. - Évolution de l'activité aldolasique

a) Chez le Lapin (•— Psoas; $\square---\square$ Soleus)

b) Chez le Poulet $(\bullet$ Pectoralis ; $\square-(-\square$ Adductors) 
L'évolution périnatale de l'activité aldolasique, glycolytique, diffère considérablement quelle que soit l'espèce étudiée entre les deux types de muscles "blancs " et " rouges" (fig. 4) :

- Dans le psoas major, muscle de type blanc chez le Lapin, l'activité enzymatique croît de façon constante et régulière ; par contre, dans le groupe des pectoralis du poulet, l'augmentation de l'activité est très rapide après la naissance : dans les muscles embryonnaires, elle se maintient à un niveau très bas, inférieur à l'activité observée dans les adductors; après la naissance et dans les io premiers jours de la vie ex ovo, on assiste à un bouleversement métabolique avec une activité glycolytique qui, dès l'âge de ro jours a atteint son niveau adulte, schéma différent de celui observé chez le Lapin où l'activité croît d'une manière régulière, et sans discontinuité apparente.

- Dans les muscles "rouges " de ces deux espèces, le schéma est différent de celui observé dans les muscles "blancs " : situé toujours à un niveau très bas par rapport à celle du psoas, l'activité aldolasique augmente relativement peu dans le soleus du lapin, particulièrement après l'âge de 15 jours. Dans le groupe des adductors du

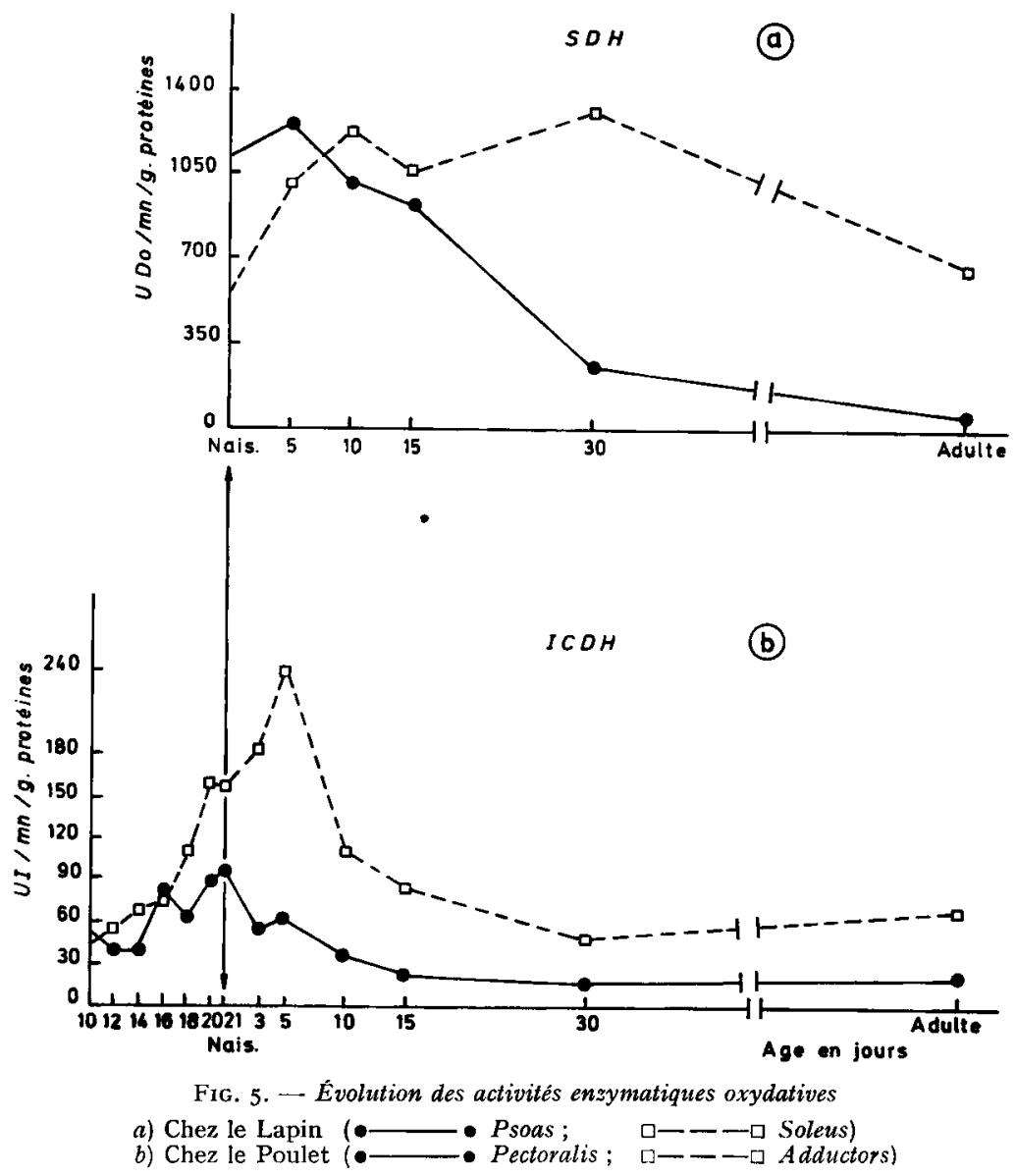


poulet par contre, on note une activité importante avant l'éclosion (double de celle des pectoralis aux mêmes âges), activité décroissant régulièrement par la suite jusqu'à l'âge de 30 jours, pour se maintenir à un niveau voisin de celui observé chez l'adulte.

L'évolution des activités enzymatiques de la voie oxydative est plus complexe :

- Dans les muscles " blancs " étudiés, les activités SDH ou ICDH sont d'abord élevées, comme l'indiquent les figures $5 a$ et $b$; ces valeurs décroissent rapidement, atteignant dès le quinzième jour chez le Poulet ou le trentième jour chez le Lapin, leur niveau adulte.

- Les muscles "rouges " ont une évolution enzymatique semblable chez le I apin et le Poulet : les maxima d'activité oxydative se situent après la naissance (un pic à 5 jours chez le Poulet, maximum étalé entre ro et 30 jours chez le Lapin). Ces activités diminuent rapidement chez le Poulet, progressivement chez le Lapin pour atteindre dans ces deux espèces chez l'adulte un taux inférieur à celui des muscles " blancs " étudiés à la naissance.

\section{DISCUSSION}

Deux faits paraissent particulièrement intéressants dans la différenciation du tissu musculaire telle que nous l'avons étudiée : d'une part, l'augmentation (soit progressive, soit rapide) du métabolisme glycolytique à partir de la naissance, d'autre part l'apparition au cours des premiers jours de la vie postnatale de maxima d'activité enzymatique, essentiellement de type oxydatif.

Au cours de la période fotale, le métabolisme musculaire semble orienté vers une activité oxydative dominante : c'est ainsi que les taux de SDH et d'ICDH sont, chez l'adulte et quelle que soit l'espèce envisagée, inférieurs ou égaux à ceux mesurés à la naissance ; pareillement, cette même activité dans les muscles " rouges " des animaux adultes est inférieure à celle mesurée dans les muscles " blancs " à la naissance, type de muscle qui chez l'adulte possède un métabolisıne à prédominance glycolytique. L'évolution de l'activité glycolytique que nous avons décrite précédemment montre, quand on la superpose à l'activité oxydative, qu'il se produit au cours du développement postnatal, sinon une inversion, tout au moins une mise en place des métabolismes musculaires parmi lesquels la voie anaérobie tend à prendre après la naissance et particulièrement dans les muscles "blancs ", une importance prépondérante.

Cette nouvelle orientation du métabolisme musculaire apparait soit progressivement, c'est le cas de l'évolution aldolasique chez le Lapin (fig. 4 a), soit rapidement chez le Poulet (fig. $4 b$ ) ; en ce qui concerne les activités oxydatives dans les deux espèces, la tendance à l'augmentation des taux enzymatiques n'est que passagère et se manifeste par l'apparition de maxima d'activité quels que soient les types de muscles considérés, ce qui semblerait montrer que, dans les 30 premiers jours de vie aérienne, l'évolution de ce métabolisme dans les divers types musculaires obéit à des systèmes semblables d'induction.

Cette hypothèse semble être apparemment en contradiction avec celle formulée 
voilà $I_{5}$ ans par BULLER et ECCLES (I960), pour lesquels la différenciation des muscles de type "rouge lent " est beaucoup plus sous la dépendance de l'innervation motrice que ne le serait celle des muscles de type "blanc rapide ". Ces auteurs s'appuient sur deux constatations : d'une part, la section de la moelle épinière chez de jeunes chatons montre qu'un muscle potentiellement lent ainsi privé d'innervation au cours de la première semaine postnatale devient presque aussi rapide qu'un muscle de type "rapide ", la même opération réalisée sur ces derniers n'affectant que très peu le développement de leurs propriétés contractiles ; d'autre part, la vitesse de contraction normalement lente des muscles crureus et soleus de ce mammifère n'est atteinte qu'après passage par une phase transitoire de contraction rapide aux environs de la quatrième semaine postnatale, alors que les muscles potentiellement rapides tels le flexor digitorus longus ou le flexor hallucis longus n'atteignent que progressivement après la naissance leur vitesse de contraction.

La comparaison de ces résultats de nature électrophysiologique avec ceux que nous avons obtenus sur le plan métabolique est donc des plus intéressante car elle montre à la fois le parallélisme existante entre vitesse de contraction et métabolisme au cours de la différenciation, ainsi que les divergences qui en résultent selon le type de tissu musculaire considéré. Ainsi, le passage par une phase de contraction rapide dans les deux premières semaines de vie des muscles potentiellement lents est semblable à l'évolution du métabolisme oxydatif au cours de la même période de ce même type de muscle; de même, l'augmentation progressive chez le Chaton, de la vitesse de contraction des muscles rapides se superpose à l'accroissement régulier de l'activité glycolytique observée dans le muscle blanc du mammifère que nous avons étudié, le Lapin. Tout semble donc se passer comme si l'évolution du type de contraction après la naissance obéissait aux mêmes facteurs que ceux induisant les transformations du métabolisme prépondérant dans l'un ou l'autre des types de muscles.

La question qui peut se poser est donc celle de la nature de ces facteurs d'induction : l'innervation motrice est un des plus importants ; toutefois, on ne sait pas encore si l'innervation agit directement par l'intermédiaire d'un ou de divers facteurs trophiques (Drachman, I967; MommaERTS, I970), ou bien si l'action est indirecte, par l'intermédiaire de l'activité motrice par exemple (GuTMann, I969; Jones et VRBOVA, I97I), ou bien encore si cette dernière n'est pas indépendante de l'action trophique nerveuse, et agissant surtout au niveau des métabolismes. Dans ce dernier cas, on pourrait penser que, parmi le ou les types de fibres musculaires initiaux $(\beta$ et $\alpha$ ), certains, au cours de la différenciation seraient plutôt sous la dépendance de l'innervation motrice, et cela pourrait être le cas des fibres $\beta$ qui forment la majeure partie des fibres d'un muscle de type "rouge lent", alors que les fibres $\alpha \mathrm{R}$ se transforment en $\alpha \mathrm{W}$, prépondérantes dans les muscles "blancs rapides " sous l'influence de l'activité motrice (AshmoRe et al., I972). Ceci expliquerait pourquoi on n'observe des transformations importantes dans le métabolisme musculaire qu'après la naissance d'une part, et d'autre part permettrait de comprendre les évolutions métaboliques différentes des types de muscle que nous avons étudiés. Un problème demeure toutefois entier : les types de fibres musculaires ne se différencient-ils que sous l'action de leur innervation motrice ou de facteurs semblables, ou bien sont-ils génétiquement déterminés, les autres facteurs ne faisant que catalyser ce qui est potentiellement inscrit dans le génôme? C'est dans ce sens que 
nous poursuivons nos travaux, une première approche (VIGNERon et al., sous presse) semblant montrer l'existence de deux populations de myoblastes au cours des stades précoces de la myogenèse.

\title{
Rę̧u pour publication en décembre 1975.
}

\section{SUMMARY}

\author{
PERINATAL DEVELOPMENT OF GLYCOLYTIC \\ AND OXIDATIVE METABOLISMS IN DIFFERENT TYPES \\ OF SKFLETAL, MUSCLES OF RABBIT AND CHICKEN
}

\begin{abstract}
Anaerobic and oxidative metabolisms are studied in different types of rabbit and chick muscles. Three main types of fibers $(\beta R, \alpha \mathrm{R}$ and $\alpha \mathrm{W})$ are differentiated and the enzymatic characteristics of a given muscle studied. During myogenesis in these animals, aerobic " white "muscle metabolism at birth evolves into anaerobic metabolism in adulthood. Such transformations do not occur in " red " muscle metabolism which is aerobic.

The complexity of the enzymatic development of anaerobic and oxidative metabolisms varies with the species or muscle type studied, i.e. aldolase activity of white muscles increases slowly in rabbits and quickly in chicks, whereas it remains small and stationary in red muscles. Aerobic enzyme activity reaches a maximum during the 30 days after birth in both the species and in all muscle types studied.
\end{abstract}

\section{RÉFÉRENCES BIBLIOGRAPHIQUES}

Adlard B. P. F., Dobbing J., I97I. Vulnerability of developing brain. 3. Development of four enzymes in the brains of normal and undernourished rats. Brain. Res., 28, 97-107.

Ashmore C. R., Tompkins G., Doerr L., I972. Postnatal development of muscle fiber types in domestic animals. J. Anim. Sci., 34, 37-4I.

Buller A. J., Eccles J. C., Eccles R. M., I 960 a. Differentiation of fast and slow muscles in the cat hind limb. J. Physiol., 150, 399-4I6.

Buller A. J., Eccles J., Eccles R. M., I96o $b$. Interactions between motoneurones and muscles in respect of the characteristic speeds of their responses. J. Physiol., 150, 417-439.

Buller A. J., Lewis D. M., r965. The rate of tension development in isometric tetanic contractions of mammalian fast and slow skeletal muscle. J. Physiol., 176, 337-354.

Crose R., I964. Dynamic properties of fast and slow skeletal muscles of the rat during development. J. Physiol., 173, 74-95.

Cosmos E., Ig66. Enzymatic activity of differentiation muscle fiber. I. Development of phosphorylase in muscles of the domestic fowl. Develop. Biol., 13, 163-181.

Cosmos E., 1970. in E. J. Briskey, R. G. CASSENS, B. B. MARSh, The physiology and biochemistry of muscle as a food, 2, Wisconsin Press.

Dawson D. M., Romanul F. C. A., 1964. Enzymes in muscles. 2. Histochemical and quantitative studies. Arch. Neurol., 11, 369-378.

Drachman D. B., I 967 . Is acetylcholine the trophic neuromuscular transmitter? Arch. Neurol., 17, 206-218.

Duвowitz V., I967. Cross-innervated mammalian skeletal muscle : histochemical, physiological and biochemical observations. J. Physiol., 193, 481-496.

Gutmann E., I969. The trophic function of the nerve cell. Scientia., 104, I22-14I.

Jones R., VRbova G., I97I. Activity as a factor for the development of some pharmacological properties of the neuromuscular function. J. Physiol., 214, 17-18.

Mommaerts W. F. H. M., Igjo. in E. J. Briskey, R. G. Cassens, B. B. Marsh, The physiology and biochemistry of muscle as a food. 2, Wisconsin Press.

Pette D., I97I. Metabolism differentiation of distinct muscle types at the level of enzymatic organisation. Adv. exp. med. biol., 11. 
PrewitT M. A., SAlafSky B., I967. Effect of cross innervation on biochemical characteristics of skeletal muscles. Am. J. Physiol., 213, 295-300.

Romanul F. C. A., Van der Meulen J. P., ig67. Slow and fast muscles after cross innervation. Enzymatic and physiological changes. Avch. Neurol., 17, 387-402.

Romanul F. C. A., I97I. Reversal of enzymatic profiles and capillary supply of muscle fibers in fast and slow muscles after cross innervation. Adv. exp. med. biol., 11.

Slater E. C., Bonner. W. D., I952. The effect of fluoride on the succinic oxidase system. Biochem. J., 52, I 85-196.

VIgneron P., Bacov F., Ashmore C. R., I976. Distribution heterogeneity of muscle fiber types in the rabbit longissimus dorsi. J. Anim. Sci. (sous presse). 
PLANCHE A

Muscle Accessorius latissimi dorsi de lapin $(2,5 \mathrm{~kg})$

Révélation (I) de l'activité ar'énosine triphosphatasique (ATPase) myofibrillaire à $\mathrm{pH} \mathrm{9,4} \mathrm{et} \mathrm{(2)} \mathrm{de} \mathrm{la} \mathrm{succinodeshydrogé-}$ nase (SDH).

- Les fibres $\beta \mathrm{R}$ (lentes oxydatives) sont ici SDH + ATPase -

- Les fibres $\alpha$ (rapides) sont:

$$
\begin{aligned}
& \text { ATPase +, SDH + }(\alpha \mathrm{R}) \\
& \text { ATPase +, SDH - }(\alpha W) .
\end{aligned}
$$



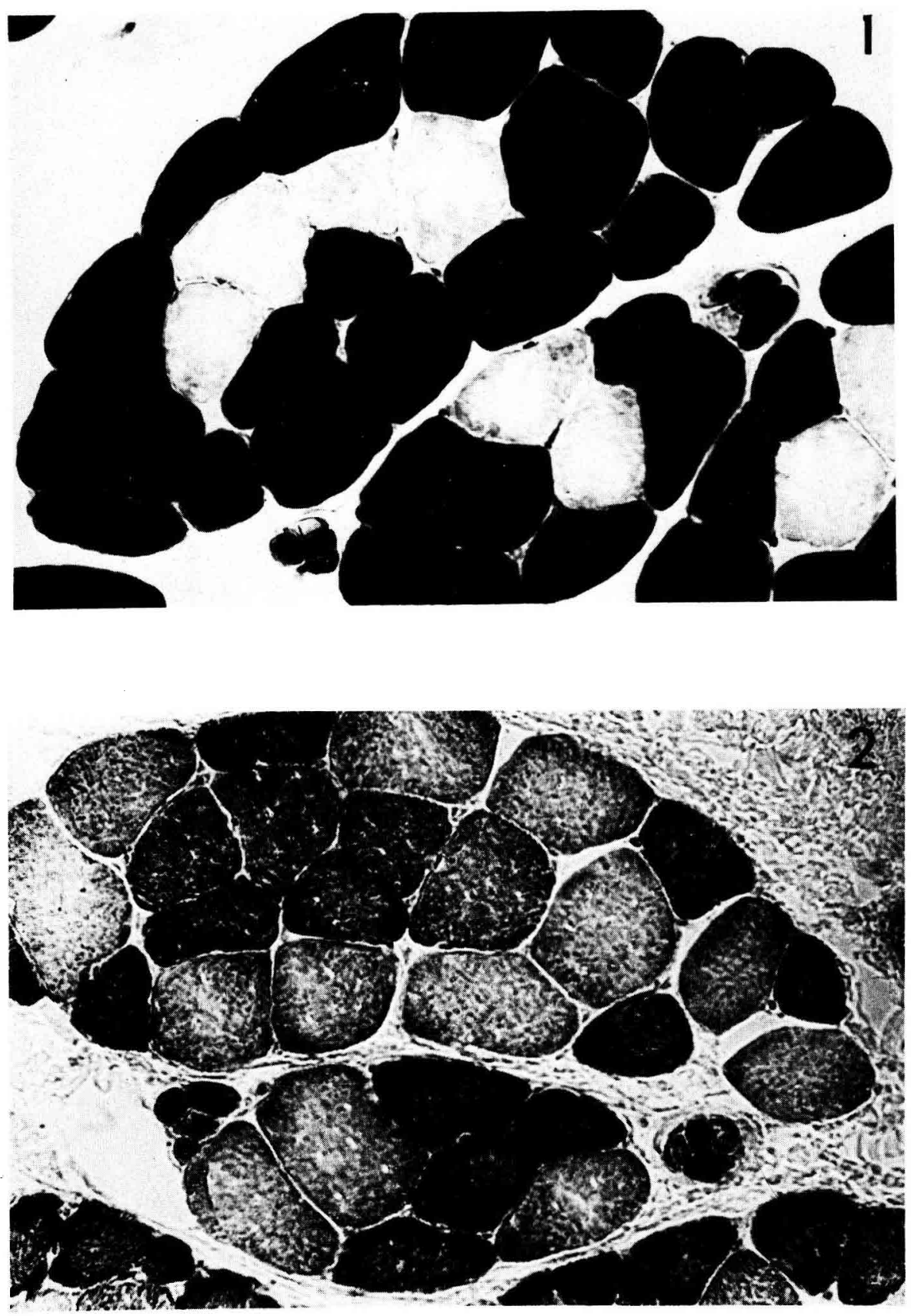

F. BACOU et P. VIGNERON 